\title{
VALIDACIÓN DEL MANEJO INTEGRADO DE Hypothenemus hampei FERRARI (COLEOPTERA: CURCULIONIDAE) EN EL HUILA, COLOMBIA
}

\author{
Ferney López Franco*, Laura Alexandra Laiton Jiménez*, Pablo Benavides Machado*
}

\begin{abstract}
LÓPEZ F., F.; LAITON J., L. A.; BENAVIDES M., P. Validación del manejo integrado de Hypothenemus hampei Ferrari (Coleoptera: Curculionidae) en el Huila, Colombia. Revista Cenicafé 70(2):91-97. 2019
\end{abstract}

El café es el principal producto agrícola de exportación de Colombia, siendo el Huila el mayor proveedor actual. La plaga más limitante es la broca de café. El objetivo de este trabajo fue validar en el campo las estrategias de control usadas en el manejo integrado de la broca. Para esto, se tomó un lote de café en el municipio de Pitalito y se dividió en cuatro parcelas experimentales, correspondiendo a los siguientes métodos de control: M1 Control biológico, M2 Control químico, M3 Control cultural y M4 Testigo manejo implementado por el agricultor. Se evaluó la infestación de broca en el campo y en café pergamino seco, por medio de un muestreo aleatorio simple. Mediante el estimador de razón de los frutos infestados por cada 100 frutos se determinó que el M3 Control cultural fue descriptivamente menor con respecto a los demás métodos de control en el campo. De manera consecuente, el M3 presentó el menor porcentaje de infestación en café pergamino seco. Cuando se implementó el control cultural, se registró en el campo una reducción del 74,55\% en el estimador de razón y del $54 \%$ en el porcentaje de infestación en café pergamino, con respecto al M4 manejo del agricultor. Esta investigación permite recomendar el control cultural como la mejor estrategia de control de la broca, así mismo, transferir estos resultados a los caficultores de Huila. El control cultural consistió en la cosecha oportuna y en el repase con recolección de frutos del suelo asistidas con dispositivos "canastillas".

Palabras clave: Control de plagas, control cultural, control biológico, control químico, manejo integrado de plagas.

\section{VALIDATION OF INTEGRATED PEST MANAGEMENT FOR COFFEE BERRY BORER, Hypothenemus hampei FERRARI (COLEOPTERA: CURCULIONIDAE) IN HUILA}

Coffee is the main exported agricultural product of Colombia, and Huila is currently the largest provider. The most limiting insect pest is Coffee Berry Borer. The objective of this study was to validate, in field conditions, the control strategies used for the Integrated Pest Management for Coffee Berry Borer. Thus, a coffee lot in the municipality of Pitalito was taken and divided into four experimental plots for the following control methods: M1 Biological control, M2 Chemical control, M3 Cultural control and M4 Control implemented by the farmer. Coffee Berry Borer infestation levels in field and in parchment coffee during harvesting was evaluated through a simple random sampling procedure. A ratio estimator of infested berries per 100 berries, the M3 cultural control was found to be descriptively lower than the other field control methods. Consequently, M3 also showed the least infested coffee parchment levels. When cultural control was implemented, there was a reduction of $74.55 \%$ on the ratio estimator and $54 \%$ on the infestation level of coffee parchment with respect to M4 Control implemented by the farmer. This research allowed to recommended cultural control as the best strategy to control Coffee Berry Borer and, ideally, to transfer these results to coffee growers in Huila. The cultural control strategy consisted in rigorous harvesting complemented with repassing and picking coffee berries from the trees and the ground using "canastilla" devices.

Keywords: Insect pest control, Cultural control, Biological control, Chemical control, Integrated Pest Management.

\footnotetext{
*Asistente de Investigación, Asistente de Investigación e Investigador Científico III, respectivamente. Disciplina de Entomología, Centro Nacional de Investigaciones de Café, Cenicafé. Manizales, Caldas, Colombia.
} 
La broca del café, Hypothenemus hampei (Ferrari) (Coleoptera: Curculionidae), es la plaga más limitante de este cultivo en el mundo (Decazy, 1987). Desde su llegada a Colombia en 1988, se ha convertido en el principal problema fitosanitario del cultivo del café por atacar y reproducirse en los frutos, ocasionando pérdidas en producción y calidad, lo que a su vez, incrementa los costos y reduce el precio de venta (Benavides et al., 2003). El departamento del Huila es el mayor productor de café de Colombia, con cerca de 150.000 hectáreas sembradas (Federación Nacional de Cafeteros de Colombia, 2018) y, por ende, la presencia de la broca en los cultivos vulnera la economía del departamento.

Esta plaga presenta dificultades para su control dada su biología y comportamiento, debido a que el adulto está protegido en el interior de los frutos y se reproduce rápidamente (Benavides et al., 2003). En este sentido, el Centro Nacional de Investigaciones de CaféCenicafé implementó un programa de Manejo Integrado de la Broca (MIB) que, además de controlar la plaga, pretende conservar los agroecosistemas cafeteros y sus múltiples interacciones (Bustillo et al., 1998); este programa combina prácticas agronómicas (Bustillo-Pardey, 2007), biológicas (Baker, 1999; Bustillo y Posada, 1996; Flórez et al., 1997), culturales (Benavides et al., 2003; Cárdenas, 1996) y químicas, tendientes a reducir las poblaciones de la broca a niveles que no causen daños económicos (Arcila, 2016; Bustillo et al., 1998; Villalba-Gault et al., 1995). Con ello, se busca mejorar la producción y la competitividad para la exportación y, además, aportar a la sostenibilidad de la producción cafetera, sin deteriorar la calidad del café y sin poner en riesgo la salud humana ni el equilibrio ambiental (Duque, 2004).

Dentro del MIB se considera como pilar fundamental el control mediante prácticas culturales, que consisten en la recolección oportuna de frutos maduros y el repase ( $\mathrm{Re}$ -Re), el cual se define como la recolección de frutos de café secos, sobremaduros y maduros de los árboles, y si es necesario frutos del suelo, finalizados los períodos de las cosechas principal y de mitad de año o “mitaca" (Benavides y Constantino, 2008). Para esta práctica, Cenicafé evaluó la eficacia de un dispositivo tipo canastilla que recolecta frutos que se encuentran en el suelo, con el que se obtuvo hasta $50,6 \%$ de reducción en los niveles de infestación de broca, respecto a un testigo donde no hubo recolección (Constantino, 2018; Constantino et al., 2017; Constantino et al., 2016). En el área del control biológico, el uso del hongo entomopatógeno Beauveria bassiana (Balsamo) Vuillemin como insecticida biológico ha demostrado ser eficaz, regulando las poblaciones del insecto, logrando hasta $70 \%$ de mortalidad en el campo (Benavides et al., 2012; Cárdenas-Ramírez et al., 2007; Góngora, 2011; Góngora-Botero et al., 2009; Jaramillo et al., 2015). El uso de insecticidas químicos es una opción dentro del MIB y su recomendación se da en función de la eficacia en el control del insecto; se ha verificado la validez de los insecticidas independiente de su formulación, ya que sólo son eficaces en el control de la broca cuando el insecto se encuentra penetrando los frutos (BustilloPardey, 2007). Dentro de los insecticidas de síntesis química usados para el control de la broca, Voliam Flexi ${ }^{\circledR}$ (100 g clorantraniliprole y 200 g tiametoxam) que contiene diamidas antranílicas, es un producto de clasificación toxicológica III (Instituto Colombiano Agropecuario - ICA, 2019) que ha mostrado porcentajes de mortalidad superiores al $85 \%$ en el campo, posterior a ocho días después de la aplicación (Arcila et al., 2013).

Dada la vulnerabilidad de los cafetales del departamento del Huila, el objetivo de este estudio fue validar en el campo estrategias 
de control usadas en el Manejo Integrado de la Broca (MIB), comparándolas con la estrategia de control realizado por un caficultor en Pitalito, Huila.

\section{MATERIALES Y MÉTODOS}

El estudio se realizó entre enero y diciembre de 2017, en la vereda Solarte, en el municipio de Pitalito (Huila), a una altitud de $1.280 \mathrm{~m}$ (150'34.8" latitud N y -76²'23.9" longitud O). Se seleccionó un lote de café variedad Castillo ${ }^{\circledR}$ con cuatro años de edad, dividido en cuatro parcelas experimentales, con 2.200 árboles cada una. En cada parcela se hizo un repase inicial, recolectando los frutos pintones, maduros, sobremaduros y secos que quedaron después de la cosecha, y posteriormente, se asignaron los siguientes métodos de control:

M1. Manejo integrado con énfasis en el control biológico: se utilizó el hongo Beauveria bassiana (Brocaril 2,0 $\mathrm{g} \mathrm{L}^{-1}$ de agua) en una concentración de $2 \times 10^{10}$ esporas por litro de agua, haciendo la aspersión con bomba de espalda y aplicando $50 \mathrm{~mL}$ de mezcla por árbol.

M2. Manejo integrado con énfasis en el control químico: se usó el insecticida Voliam Flexi ${ }^{\circledR}$ utilizando $1,4 \mathrm{~mL} \mathrm{~L}^{-1}$ de agua y $50 \mathrm{~mL}$ de la mezcla por árbol.

M3. Manejo integrado con énfasis en el control cultural: en este esquema de manejo integrado se eliminaron las ramas bajas de los árboles correspondientes al $20 \%$ del área foliar después del repase inicial. Adicionalmente, se recolectaron frutos del suelo usando dispositivos de asistencia manual (canastilla).

M4. Manejo del agricultor: se realizó un seguimiento al manejo de la broca según el criterio del agricultor, el cual consistió en cosechas oportunas.
Para cada método de control evaluado se determinó la manera y la frecuencia de aplicación, utilizando la metodología desarrollada por Benavides et al. (2003), la cual consistió en realizar unas evaluaciones diagnóstico, que incluyeron el registro semanal de las floraciones para determinar el período crítico de la broca, la estimación del porcentaje de infestación de la plaga en el campo y el análisis de la posición del insecto en el fruto. De esta manera, se controló la plaga en M1 y M2 cuando se presentaron las siguientes tres condiciones: (1) los frutos estaban en período crítico, es decir, entre los 120 y 150 días después de la floración principal, (2) el porcentaje de infestación de broca superó el $2 \%$, y (3) el porcentaje de broca encontrada en posiciones A y B fue mayor al $50 \%$.

Infestación de broca en el campo. En cada parcela se evaluaron mensualmente 60 árboles de café seleccionados por medio de un muestreo aleatorio simple, en los cuales se procedió a tomar la información de la siguiente manera: se seleccionó la rama con mayor producción, donde se contabilizaron el número de frutos totales (FT) y aquellos que se encontraban perforados por la broca (FB), con el fin de estimar el porcentaje de infestación. Se realizó un análisis descriptivo de la información utilizando el promedio del porcentaje de infestación y un estimador de razón (Ecuación $<1>$ ) por cada método de control y cada mes, teniendo en cuenta solo la información de los árboles con más de 20 frutos totales en cada evaluación.

$$
\text { Estimador de razón }=\left(\frac{F B}{(F T-F B)}\right) * 100 \quad<1>
$$

Infestación de broca en el café pergamino seco (c.p.s.). En tres pases de la cosecha principal, ocurridos en septiembre y octubre del 2017, se beneficiaron tres muestras de $15 \mathrm{~kg}$ de café 
cereza por parcela. Posteriormente, se evaluó el nivel de infestación de broca en el pergamino seco resultante con ayuda de la Cooperativa de Caficultores del Huila- Cadefihuila de Pitalito. Se realizó un análisis descriptivo estimando el promedio del porcentaje de infestación y el rango para cada método de control.

\section{RESULTADOS Y DISCUSIÓN}

\section{Infestación de broca en el campo}

El método de control que presentó los menores porcentajes de infestación durante todo el período de evaluación fue el M3 Cultural (Tabla 1). En casi todos los meses los porcentajes de infestación de broca no superaron el 4\%; sin embargo, en noviembre, finalizando la cosecha se presentaron los índices más altos de infestación, siendo el M3 Cultural con
$2,71 \%$, el método de control que presentó el menor porcentaje de infestación.

De manera descriptiva, a través del estimador de razón, fue posible determinar que en el mes de noviembre el M3 Cultural presentó el menor número de frutos brocados por cada 100 frutos, mostrando una reducción del 74,55\% con respecto al M4 Manejo del agricultor; este valor es superior al reportado por Constantino et al. (2017), que encontraron 50,6\% menos infestación con respecto a un testigo sin repase. Así mismo, el M2 Químico y el M1 Biológico tuvieron una disminución de $61,49 \%$ y $49,46 \%$, respectivamente (Figura 1), siendo el primero similar al reportado por Arcila et al. (2013), quienes obtuvieron una reducción del 64\% en la infestación de broca con respecto a un testigo en pruebas de campo cuando aplicaron insecticidas de síntesis química.

Tabla 1. Porcentaje de infestación de la broca en el campo por método de control (promedio y error estándar).

\begin{tabular}{|c|c|c|c|c|}
\hline Mes & M1 Biológico & M2 Químico & M3 Cultural & M4 Manejo del agricultor \\
\hline Enero & $0,37 \pm 0,20$ & $0,12 \pm 0,05$ & $0,17 \pm 0,09$ & $0,33 \pm 0,13$ \\
\hline Febrero & $1,27 \pm 0,28$ & $1,42 \pm 0,34$ & $0,6 \pm 0,25$ & $1,95 \pm 0,28$ \\
\hline Marzo & $1,51 \pm 0,23$ & $0,95 \pm 0,16$ & $0,53 \pm 0,12$ & $0,92 \pm 0,13$ \\
\hline Abril & $1,44 \pm 0,27$ & $2,73 \pm 0,40$ & $0,7 \pm 0,14$ & $2,14 \pm 0,44$ \\
\hline Mayo & $2,66 \pm 0,52$ & $1,47 \pm 0,30$ & $0,89 \pm 0,31$ & $3,19 \pm 0,45$ \\
\hline Junio & $2,53 \pm 0,38$ & $1,73 \pm 0,30$ & $0,62 \pm 0,14$ & $2,1 \pm 0,43$ \\
\hline Julio & $0,79 \pm 0,16$ & $1,88 \pm 0,41$ & $0,92 \pm 0,52$ & $1,28 \pm 0,25$ \\
\hline Agosto & $2,28 \pm 0,43$ & $2,48 \pm 0,45$ & $1,03 \pm 0,21$ & $3,83 \pm 0,70$ \\
\hline Septiembre & $1,62 \pm 0,31$ & $1,52 \pm 0,30$ & $1,28 \pm 0,32$ & $2,46 \pm 0,46$ \\
\hline Octubre & $2,02 \pm 0,34$ & $2,63 \pm 0,53$ & $1,47 \pm 0,32$ & $2,77 \pm 0,49$ \\
\hline Noviembre & $12,08 \pm 2,76$ & $7,31 \pm 1,50$ & $2,71 \pm 0,62$ & $15,65 \pm 2,19$ \\
\hline Diciembre & $0 \pm 0,00$ & $0 \pm 0,00$ & $0 \pm 0,00$ & $0 \pm 0,00$ \\
\hline Promedio (año) & $2,38 \pm 0,91$ & $2,02 \pm 0,54$ & $0,91 \pm 0,20$ & $3,86 \pm 1,98$ \\
\hline
\end{tabular}


Infestación de broca en el café pergamino seco (c.p.s.)

En la evaluación de infestación de broca en el c.p.s., descriptivamente, el método de control que presentó el menor porcentaje de infestación fue el M3 Cultural (Figura 2), seguido del M2 Químico con un $0,86 \%$. El método de control con mayor porcentaje de infestación fue el M4 Manejo del agricultor con 1,39\%.

El método M3 Cultural presentó una mayor reducción en el porcentaje de infestación de broca con $54 \%$ con respecto a M4 Manejo del agricultor. El M2 Químico y el M1 Biológico disminuyeron $38 \%$ y $22 \%$, respectivamente. Los resultados del M3 Cultural superaron los valores de $28 \%$ reportados por Constantino et al. (2017) cuando fue comparado frente a un testigo sin repase. E1 M2 Químico mostró un resultado inferior al obtenido por Arcila et al. (2013) de 66\% de la infestación en el c.p.s. al usar productos organofosforados.

Si bien la literatura reporta que existen expresiones matemáticas que relacionan la infestación en el campo con aquella en el café pergamino seco (Montoya, 1999), estas funciones no aplican para esta investigación dado que la evaluación del café pergamino seco correspondió a tres pases de cosecha principal, ocurrida entre los meses de septiembre y octubre del 2017. La información del mes de noviembre se limita al muestreo de campo.
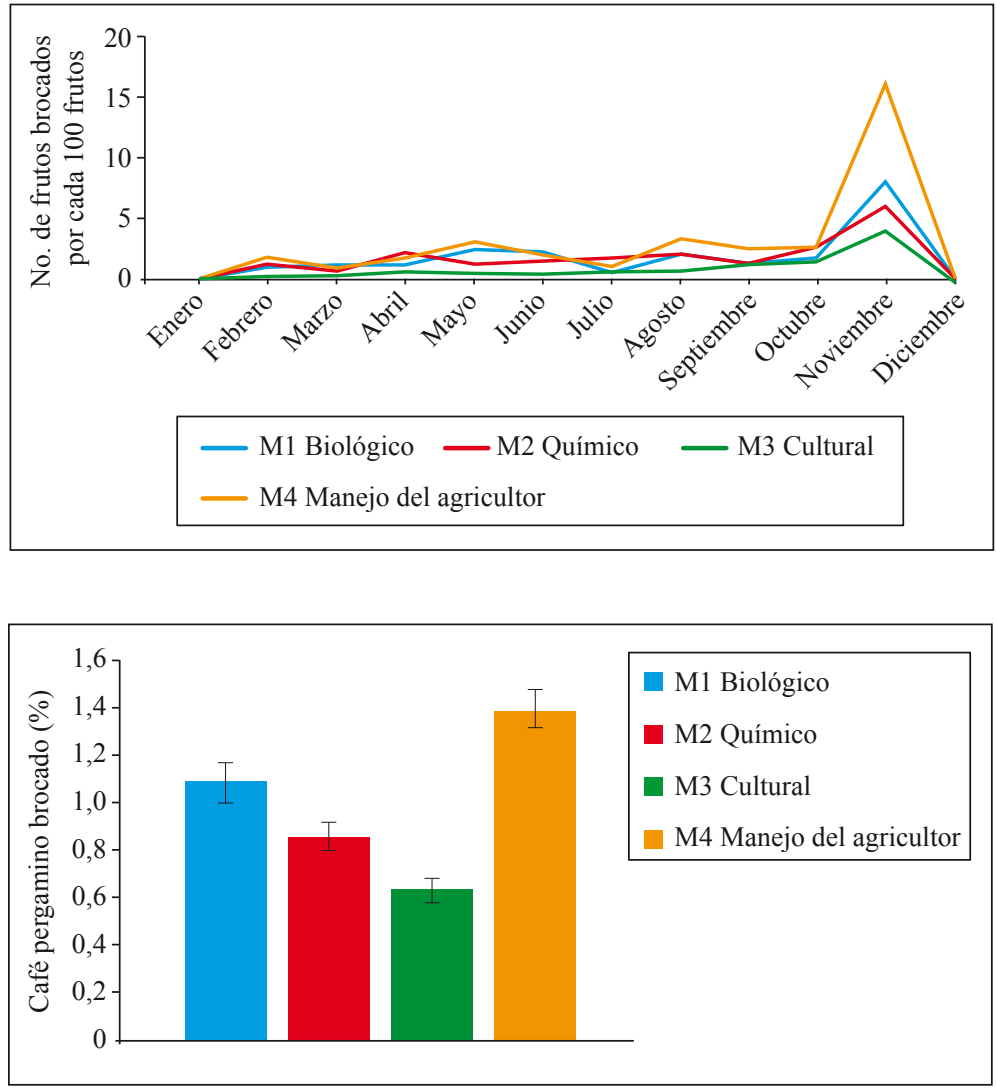

Figura 1. Estimador de razón para el número de frutos brocados por cada 100 frutos a través del tiempo para cada método de control.
Figura 2. Promedio del porcentaje de café pergamino seco brocado para cada método de control. Las barras indican el rango. 
Sin embargo, como era de esperarse, en los métodos donde se encontraron las mayores cantidades de frutos infestados en el campo, también se observaron las mayores infestaciones en el café pergamino seco.

En este estudio puede concluirse que el manejo integrado de la broca disminuye y mantiene los niveles de la broca por debajo del daño económico. De todos los métodos de control utilizados dentro del MIB, el énfasis con control cultural presentó los mejores resultados; siendo la recolección del suelo con "canastillas" una práctica viable económicamente y con impacto en la disminución de la broca.

\section{AGRADECIMIENTOS}

Este estudio hizo parte del proyecto de investigación ENT107006, financiado con recursos del Sistema General de Regalías del departamento de Huila (Colombia), mediante el convenio CN-2015-0025. Los autores agradecen a la auxiliar de investigación Adriana Paola Gómez por el apoyo en las labores de campo y a la Cooperativa de Caficultores Cadefihuila de Pitalito (Huila) por su apoyo en las evaluaciones de calidad física del café.

\section{LITERATURA CITADA}

Arcila, A. (2016). Insecticidas químicos recomendados para el control de la broca del café. Brocarta, 49, 2.

Arcila, A., Duarte, A. F., Villalba, D. A., \& Benavides, P. (2013). Nuevo producto en el manejo integrado de la broca del café en Colombia. Avances Técnicos Cenicafé, 437,1-8. Recuperado de http://hdl.handle.net/10778/477

Baker, P. S. (1999). La broca del café en Colombia; Informe final del proyecto MIP para el café DFID-Cenicafé-CABI Bioscience. Chinchiná, Colombia: Cenicafé

Benavides, P., Bustillo, A. E., Cárdenas, R., \& Montoya., E. C. (2003). Análisis biológico y económico del manejo integrado de la broca del café en Colombia. Revista Cenicafé, 54 (1), 5-23.

Benavides, P., \& Constantino, L. M. (2008). Control cultural en el manejo integrado de plagas. En A. E. Bustillo P. (Ed.), Los insectos y su manejo en la caficultura colombiana (pp. 234 - 241). Manizales, Colombia: Cenicafé.

Benavides, P., Góngora, C., \& Bustillo, A. (2012). IPM Program to Control Coffee Berry Borer Hypothenemus hampei, with Emphasis on Highly Pathogenic Mixed Strains of Beauveria bassiana, to Overcome Insecticide Resistance in Colombia. En F. Perveen(Ed.), Insecticides-Advances in Integrated Pest Management (pp. 511-540). https://doi.org/10.5772/28740

Bustillo, A. E., Cárdenas, R., Villalba, D. A., Benavides, P., Orozco, J., \& Posada, F. J. (1998). Manejo integrado de la broca del café: Hypothenemus hampei Ferrari en Colombia. Recuperado de http://biblioteca.cenicafe.org/ handle/10778/848

Bustillo, A. E., \& Posada, F. J. (1996). El uso de entomopatógenos en el control de la broca del café en Colombia. Manejo Integrado de Plagas, Costa Rica, 42, 1-13.

Bustillo-Pardey, A. E. (2007). El manejo de cafetales y su relación con el control de la broca del café en Colombia. Boletín técnico Cenicafé, 24, 1-40. Recuperado de http:// hdl.handle.net/10778/579

Vélez, P.E., Posada, F.J., Marín, P., González, M.T., Osorio, E. \& Bustillo, A.E. (1997). Técnicas para el control de calidad de formulaciones de hongos entomopatógenos. Boletín técnico Cenicafé, 1-37. Recuperado de http:// hdl.handle.net/10778/709

Cárdenas M., R. (1996). Control cultural de la broca del café. En Memorias del Curso de Actualización sobre Manejo Integrado de la Broca del Café (Comité Departamental de Cafeteros de Antioquia-SIADA-Cenicafé). Medellín, Colombia.

Cárdenas-Ramírez, Á. B., Villalba-Guott, D. A., BustilloPardey, A. E., Montoya-Restrepo, E., \& Góngora, C. (2007). Eficacia de mezclas de cepas del hongo Beauveria bassiana en el control de la broca del café. Revista Cenicafé, 58(4), 293-303.

Constantino, L. M. (2018). Impacto del repase en la cosecha principal de café. Brocarta, 50, 2.

Constantino, L. M., Oliveros, C. E., Benavides, P., Gómez, J. C., Serna, C. A., Ramírez, C. A., Arcila, A. (2016). 
Recolección de frutos de café del suelo con canastilla: Herramienta para el manejo integrado de la broca. Avances Técnicos Cenicafé, 468, 1-8. Recuperado de http://hdl.handle.net/10778/706

Constantino, L. M., Oliveros, C. E., Benavides, P., Serna, C. A., Ramírez, C. A., Medina, R. D., \& Arcila, A. (2017). Dispositivo recolector de frutos de café del suelo para el manejo integrado de la broca. Revista Cenicafé, 68 (1), 22-37.

Decazy, B. (1987). Métodos de control químico y cultural de la broca del fruto de cafeto. En Memorias del curso sobre manejo integrado de plagas del cafeto con énfasis en broca del fruto Hypothenemus hampei (Ferrari) (pp. 147-158). Guatemala: IICA: PROMECAFE.

Duque, H. (2004). Cómo reducir los costos de producción en la finca cafetera. (2. ${ }^{\mathrm{a}}$ ed.). Recuperado de https:// www.cenicafe.org/es/publications/Como_reducir_ los_costos_de_producci\%C3\%B3n_en_la_finca_ cafetera.pdf

Federación Nacional de Cafeteros de Colombia-FNC. (2018). Informe del gerente general 2018. Recuperado 24 de abril de 2019, de https://www.federaciondecafeteros. org/static/files/Periodico_IGG2018.pdf

Flórez, E., Bustillo, A. E., \& Montoya, E. C. (1997). Evaluación de equipos de aspersión para el control de Hypothenemus hampei con el hongo Beauveria bassiana. Revista Cenicafé, 48 (2), 92-98.
Góngora, C. E. (2011). ¿Cómo usar el hongo Beauveria bassiana para proteger su cosecha de café?. Brocarta, 42,2 .

Góngora-Botero, C. E., Marín-Marín, P., \& BenavidesMachado, P. (2009). Claves para el éxito del hongo Beauveria bassiana como controlador biológico de la broca del café. Avances Técnicos Cenicafé, 384, 1-8.

Instituto Colombiano Agropecuario - ICA. (2019). Listado de registros nacionales de plaguicidas químicos de uso agrícola. Recuperado de https://www.ica.gov.co/getdoc/ d3612ebf-a5a6-4702-8d4b-8427c1cdaeb1/registrosnacionales-pqua-15-04-09.aspx

Jaramillo, J. L., Montoya, E. C., Benavides, P., \& Góngora, C. E. (2015). Beauveria bassiana and Metarhizium anisopliae mix to control the coffee berry borer in soil fruits. Revista Colombiana de Entomología, 41(1), 95-104.

Montoya, E. C. (1999). Caracterización de la infestación del café por la broca y efecto del daño en la calidad de la bebida. Revista Cenicafé, 50(4), 245-258.

Villalba-Gault, A., Bustillo-Pardey, A. E., \& ChavesCórdoba, B. (1995). Evaluación de insecticidas para el control de la broca del café en Colombia. Revista Cenicafé, 46(3), 152-163. 


\section{FEDERACIÓN NACIONAL DE CAFETEROS DE COLOMBIA GERENCIA TÉCNICA \\ PROGRAMA DE INVESTIGACIÓN CIENTÍFICA \\ Centro Nacional de Investigaciones de Café \\ "Pedro Uribe Mejía"}

\section{DIRECCIÓN}

Álvaro León Gaitán B., Microbiólogo, Ph.D.

Ángela Jaramillo G., Prof. en Comercio Internacional, Esp.

\section{DISCIPLINAS DE INVESTIGACIÓN}

\section{Calidad}

Valentina Osorio P., Ing. Alimentos, Esp.

Jenny Paola Pabón U., Ing. Agrícola, M.Sc.

Claudia Patricia Gallego A., Bacterióloga

Luz Fanny Echeverry G., Química

Claudia Rocío Gómez P., Tecnóloga Química

Alexis Urriago Y., Técnico en Producción Agrícola

Paola Andrea Calderón T., Tecnólogo en Producción

Agropecuaria Ecológica

\section{Biometría}

Rubén Darío Medina R., Estadístico, M.Sc.

Esther Cecilia Montoya R., Estadístico, M.Sc.

Hernando García O., Ing. Agroforestal

Luis Carlos Imbachi Q., Estadístico

Nancy Flórez P., Aprendiz

\section{Economía Agrícola}

Hugo Mauricio Salazar E., Ing. Agrónomo, M.Sc.

César Alberto Serna G., Contador, M.Sc.

Juan Carlos Gómez S., Contador, Ing. Agrónomo, M.Sc

Fisiología Vegetal

José Ricardo Acuña Z., Biólogo Ph.D.

Aristófeles Ortiz, Químico, M.Sc.

Carlos Andrés Unigarro M., Ing. Agrónomo, M.Sc.

Natalia Catalina Flechas B., Bióloga

Marta Bibiana Escobar P., Tecnóloga Química

Claudia Patricia Valencia V. Técnico en producción de café

Claudia Marcela Mora A.

Mario Franco A.

Juan David Osorio G., Pasante Universitario

\section{Suelos}

Siavosh Sadeghian K., Ing. Agrónomo, Ph.D. Hernán González O., Ing. Agrónomo, Ph.D.

Luis Fernando Salazar G., Ing. Agrónomo, Ph.D.

Luz Adriana Lince S., Ing. Agrónomo, Geóloga, M.Sc.

Juan Camilo Rey S., Ing. Agrónomo. M.Sc.

Vanessa Catalina Díaz P., Química, Esp

Stephania Rincón B., Tecnóloga Química

Sebastián García B. Tecnólogo en Gestión de Recursos

Naturales

Arturo Gómez V.

\section{Fitotecnia}

Francisco Fernando Farfán V., Ing. Agrónomo, M.Sc. José Raúl Rendón S., Ing. Agrónomo, M.Sc.
Andrés Mauricio López L., Médico Veterinario y Zootecnista, M.Sc.

Rocío Espinosa A., Médico Veterinario y Zootecnista, M.Sc. Andrés Felipe León B., Ing. Agrónomo Cristian Salomón Rodríguez V., Ing. Agrónomo Alexander Jaramillo J.

\section{Mejoramiento Genético}

Claudia Patricia Flórez R., Ing. Agrónomo, Ph.D. Diana María Molina V., Bacterióloga, Ph.D.

Carlos Ernesto Maldonado L., Ing. Agrónomo, M.Sc.

Juan Carlos Arias S., Ing. Agrónomo, M.Sc.

Hernando A. Cortina G., Ing. Agrónomo, M.Sc.

Julio Quiroga C., Ing. Agrónomo, M.Sc.

Luisa Fernanda López M., Ing. Agrónomo

Carlos Augusto Ramírez C., Administrador de Empresas

Agropecuarias

Carlos Augusto Vera A., Administrador Financiero

Jairo Jaramillo O., Ing. Mecatrónico

Marlio Fernando Abella D., Agrónomo

Mauricio Jiménez S., Ing. Agrónomo, M.Sc.

Gilbert Rodríguez Q., Técnico Profesional en Desarrollo de Producción Pecuaria

Jhon Esteban Quintero A., Técnico en Producción de Café Hernán Díaz C.

Cruz Elena Díaz M.

Omar Villarreal

Keiner Sebastián Jaramillo T., Pasante Universitario

Stefania Marulanda R., Pasante Universitario

\section{Entomología}

Pablo Benavides M., Ing. Agrónomo, Ph.D.

Carmenza E. Góngora B., Microbióloga, Ph.D.

Zulma Nancy Gil P., Ing. Agrónomo, Ph.D.

Marisol Giraldo J., Ing. Agrónomo, Ph.D.

Lucio Navarro E., Biólogo, Ph.D.

Luis Miguel Constantino C., Biólogo Entomólogo, M.Sc.

Aníbal Arcila M., Ing. Agrónomo

Mauricio Jiménez Q., Agrónomo

Jesús Hernando Gómez L., Biólogo

Juan Diego Maldonado C., Biólogo

Ferney López F., Ing. Agrónomo

Laura Alexandra Laiton J., Ing. Agrónomo

Diana Soraya Rodríguez A., Técnico en Producción de Café

Luis Eduardo Escobar S., Tecnólogo en Gestión

Agropecuaria

Juan Carlos Ortiz F.

Carlos Alberto Quintero A.

Claudia Bibiana Tabares B.

Faber de Los Ríos $P$.

Diana Marcela Giraldo V.

José Robín García Castaño

Fernán Santiago Mejía A., Pasante Universitario

Juan Guillermo Orrego M., Pasante Universitario 


\section{Agroclimatología}

Juan Carlos García L., Ing. Agrónomo, Ph.D.

Ninibeth Gibelli Sarmiento H., Ing. Agrícola

Carolina Ramírez C., Ing. Agrícola, M.Sc.

Jonnier Alejandro Hoyos A., Tecnólogo en gestión

agropecuaria

Wílmar A. Rendón G., Tecnólogo en Sistemas Informáticos

Jorge Wilmar Valencia G., Tecnólogo en Análisis y

Desarrollo de Sistemas de Información

Luis Gonzaga Henao R.

Luis Fernando Torres $Q$.

Orlando Salazar G.

\section{Fitopatología}

Carlos Ariel Ángel C., Ing. Agrónomo, Ph.D.

Rosa Lilia Ferrucho, Ing. Agrónoma, Ph.D.

Nancy del Carmen Arciniegas B., Ing. Agrónoma, M.Sc.

Gustavo Adolfo Marín R. Ing. Agrónomo, M.Sc.

Carlos Alberto Rivillas O., Ing. Agrónomo, M.Sc.

Isabel Cristina Ramírez P., Ing. Agrónomo

Carlos Alberto Zuluaga E., Técnico en Mantenimiento de

Computadores y Redes de datos.

Carlos Arturo González V.

Jorge Dicksson Ocampo M.

Jaroliver Cardona G.

Cindy Michel Ospina G., Aprendiz

\section{Poscosecha}

Juan Rodrigo Sanz U., Ing. Mecánico, Ph.D.

Nelson Rodríguez V., Ing. Químico, Ph.D.

Aída Esther Peñuela M., Ing. Alimentos, Ph.D.

César Augusto Ramírez G., Arquitecto, M.Sc.

Juan Carlos López N., Microbiólogo, M.Sc.

Álvaro Guerrero A., Ing. Electrónico/Electricista, M.Sc.

Laura Vanessa Quintero Y., Ing. Química, M.Sc.

Carlos Alfonso Tibaduiza V., Ing. Agrícola, M.Sc.

Diego Antonio Zambrano F., Ing. Químico

Ricardo José Grisales M., Tecnólogo en Electrónica

Jorge Alexander Londoño C., Técnico en Soldadura

Mario Espinosa G.

Javier Velásquez $\mathrm{H}$.

Samuel Castañeda

Camilo Andrés Morcillo S., Pasante Universitario

José Miguel Jaramillo G., Aprendiz Universitario

Valeria Barco G., Aprendiz

\section{EXPERIMENTACIÓN}

Carlos Gonzalo Mejía M., Administrador de Empresas

Agropecuarias, M.Sc.

José Farid López D., Tecnólogo en Administración

Agropecuaria

Alejandra Marcela Garcés G., Tecnólogo en Administración

Agropecuaria

Yolanda Castaño G.

\section{Estación Experimental Naranjal}

Jhon Félix Trejos P., Ing. Agrónomo

Tito Alberto Ramírez S.

Víctor Alfonso Díaz R., Aprendiz

\section{Estación Experimental El Tambo}

Hernán Darío Menza F., Ing. Agrónomo, M.Sc.
Estación Experimental EI Rosario

Carlos Mario Ospina P., Ing. Forestal, M.Sc.

Estación Experimental La Catalina

Melsar Danilo Santamaría B., Ing. de Alimentos

Estación Experimental La Trinidad

Myriam Cañón H., Ing. Agrónomo

Estación Experimental Paraguaicito

Daniel Antonio Franco C., Agrónomo

Estación Experimental Pueblo Bello

José Enrique Baute B., Ing. Agrónomo

Estación Experimental San Antonio

Carlos Roberto Ariza O., Ing. Agrónomo, Esp.

\section{APOYO A LA INVESTIGACIÓN}

Divulgación y Transferencia

Sandra Milena Marín L., Ing. Agrónoma, M.Sc.

Jair Montoya T., Administrador de Empresas, M.Sc.

Carmenza Bacca R., Diseñadora Visual

Óscar Jaime Loaiza E., Diseñador Visual

Luz Adriana Álvarez M., Diseñadora Visual

Paula Andrea Salgado V., Administrador Financiero

David Antonio Torres C., Aprendiz

Gineth Lorena Gaviria B., Aprendiz

Tecnología de la Información y Comunicaciones

Luis Ignacio Estrada H., Ing. Químico

Carlos Hernán Gallego Z., Ing. de Sistemas, Esp.

Miguel Alfonso Castiblanco C., Bibliotecólogo/Ing. de

Sistemas

Elkin Marcelo Valencia L., Ing. de Sistemas, Esp.

Daniel Orozco J., Ing. de Sistemas y Telecomunicaciones, Esp.

Leonardo Adolfo Velásquez N., Ing. de Sistemas y

Telecomunicaciones, Esp.

Kevin Adolfo Hincapié V., Ing. de Sistemas y

Telecomunicaciones, Esp.

Andrés Felipe Ramírez M., Ing. de Sistemas y

Telecomunicaciones, Esp.

José Rubiel Castrillón G., Ing. de Sistemas y

Telecomunicaciones, Esp.

Audberto Quiroga M., Biólogo, Esp.

Juan Camilo Espinosa O., Ing. Topográfico, Esp.

Juan Pablo Carvajal B., Ing. Electrónico

Federico Higuera R., Pasante, Universitario

Miguel Ángel Salamanca Q., Pasante Universitario

\section{UNIDAD ADMINISTRATIVA Y FINANCIERA}

Luz Miryam Corredor R., Administradora de Empresas,

Contador Público, Esp.

Rufina Perdomo G.

Nancy Elena Pérez M., Contador

\section{Gestión Contable y Tributaria}

Martha Elena Vélez H., Contadora, Esp.

Daniela López O., Contadora, Esp. 
Jorge Eduardo Dávila M., Contador Público, Esp.

Luis Alfredo Amaya F., Administrador Público

Lina Marcela Patiño G., Administradora de Empresas

Mariana Osorio L., Aprendiz

Gestión de Bienes y Servicios - Compras y Contratación

Juan David Álzate O., Economista, M.Sc.

Mauricio Loaiza M., Ing. Industrial

Carolina Correa M., Profesional en Negocios

Internacionales

Diego Alejandro Muñoz G., Economista

Lina María Giraldo., Administrador Financiero

Luz Stella Duque C., Tecnóloga en Administración de

Negocios

Jesús Alberto Vergara T., Técnico en Sistemas

Gabriel Antonio Melo P.

Laura Vanessa Hernández T., Aprendiz

Natalia Bueno V., Aprendiz

\section{Gestión de Bienes y Servicios - Mantenimiento}

Paulo Alejandro Arias C., Ing. Electricista, Esp.

Cristian Andrés Chica G., Ing. Mecatrónico

María Alejandra López R., Ing. Mecatrónica

Robinson Guzmán G., Técnico Electricista

Jorge Hernán Marulanda E., Tecnólogo en Electrónica

Albert Johanne Agudelo L., Tecnólogo en Electrónica

Gabriel Hernando Ortiz C., Tecnólogo en Gestión Bancaria

y Financiera

José Gildardo Aguirre O., Tecnólogo en Mantenimiento

Mauricio Londoño V., Tecnólogo en Mantenimiento Eléctrico Industrial
Electrónico e Instrumental Industrial

Uriel López P.

Eduardo Villegas A.

Fredy Hernán Osorio C.

Jhon Fredy Rojo G.

Jorge Eduardo Sepúlveda V.

Jorge Antonio Arias A.

Carlos Andrés Hernández G.

Fabián Sánchez L.

Mario López L.

Daniel Castaño G., Aprendiz

Gestión de Tesorería

Jesús Danilo González O., Contador, Esp.

Gestión del Talento Humano

Érica Mayerly Galvis R., Trabajadora Social, M.Sc.

Elsa Natalia Quintero C., Profesional en Salud Ocupacional, M.Sc.

Germán Uriel Granada, Administrador de Empresas, Esp. Luz Yaneth Guarín C., Tecnóloga en Administración de Negocios

\section{Planeación Financiera y Presupuesto}

Jesús Alberto Cardona L., Ing. Industrial, M.Sc., PMP® Valentina Sepúlveda C., Ingeniera Industrial, Esp., PMP® Juan Manuel Meza S., Administrador de empresas, Esp. Josué David Espitia F., Economista

Diana Marcela Buitrago R., Administrador de empresas Cindy Yohanna Salazar V., Técnica en Administración 